\title{
SECURE DATA STORAGE OVER DISTRIBUTED NODES IN NETWORK THROUGH BROADCAST TECHNIQUES
}

\author{
Prashant S. Sadaphule ${ }^{1}$, N. A. Dhawas ${ }^{2}$

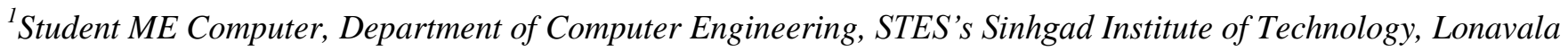 \\ Maharashtra, India \\ ${ }^{2}$ Assistant Professor, Department of Information Technology, STES's Sinhgad Institute of Technology, Lonavala \\ Maharashtra, India
}

\begin{abstract}
Abstract Network wide broadcasting is one of the major mechanisms in wireless network to resolve may issues. In wireless network communication the source node sends a message to all other nodes that are present in the network. The nodes are communicating with each other but these nodes do not have any pre-existing routing infrastructure for communication. No static infrastructure is available in wireless network communication, because of this there is limited transmission range. When source node wants to communicate with nodes that are outside the radio range of network then there is need to form a multihop network. This paper proposes secure data storage over distributed network distributed through broadcast algorithm. System manages the data security, data backup, perform faster communication in between node and reduce the collision
\end{abstract}

Keywords: Broadcast, Data Security, Distributed Node, Wireless Network.

\section{INTRODUCTION}

Today's wireless communication technology is very powerful and advance communication technology. In wireless communication there are various portable and handheld devices that made it possible to stay connected anywhere, anytime. Reliable high speed network connectivity is achieved with movable nodes and through connectivity the portable and handheld devices will do the data communication. The wireless node can communicate with each other in two fashions:

\subsection{Single Hop Fashion}

In these communications method the sender and receiver node are present in the communication range of each other so sender can directly send the message to receiver node. No need of intermediate node.

\subsection{Multi Hop Fashion}

In this case the source node sends the message to the intermediate neighbour node and the intermediate node sends the message to receiver node. In this case the source and receiver node are not present in the communication range of each other.

The interference is a major limiting factor in wireless network when two or multiple nodes transmit a message to a common neighbor node, because of collision the common node will not receive any message from any sending nodes. The transmission range causes the serious problems of redundancy, contention and collision problem. All these issues are collectively called as broadcast storm problem. To achieve the aim of reliability in broadcast mechanism, reduction in number of retransmission of messages will result in reduced contention and collision.

In this paper study of problems related to broadcasting message in wireless network is done. Various network applications use broadcast mechanism. The major requirement of these applications is collision free data delivery, low end to end latency and low redundancy. Data is maintained on various nodes through broadcasting and scheduling mechanism. For distributed data storage first step will be data broadcast from single node, and second to extend the system into data broadcast from multiple nodes to all other nodes that are available in the network.

\section{LITERATURE SURVEY}

Today's wireless communication is very powerful technology. The current state of the art of wireless networks is captured in [1], where solutions are discussed with various broadcasting schemes. Gandhi et al.[1] introduces two algorithms for broadcasting in wireless network such as ONE to ALL and ALL to ALL data broadcast. The main objective is to compute a schedule in which the latency is minimized. In the network model the interference and transmission range are identical and the interference range is strictly larger than the transmission range. The algorithm constructs the broadcast tree and then schedules the transmission, so that every node receives the message collision free. The ONE to ALL problems, a simple approximation algorithm that achieves the 12 approximation scheme. For ALL to ALL broadcasting, two algorithms by efficient scheduling scheme are designed. The first achieves an approximation guarantee of 20. The second algorithm achieves the approximation factor of 34. Gandhi et al. [2] addresses, two main issues of broadcasting such as latency 
and redundancy in ad hoc network. It gives two algorithms, first is scheduling broadcast for single message and second is scheduling broadcast for multiple messages. First algorithm proved that minimum latency collision free broadcasting in ad hoc networks is NP hard. It minimizes the broadcast latency and number of retransmissions in ad hoc network. Ho et al. [3] addresses flooding issues for multicast in multihop ad hoc networks. The main focus is on multicast routing in highly mobile ad hoc network. This is very challenging issue. For the simulation of this system, author uses the Global Mobile System Simulator (GloMoSim) v.1.1.1 developed at UCLA which has two mobility models, random waypoint and drunken mobility. The simulator were performed for 50 nodes randomly placed in 1000x1000 field using 7 different power ranges from 100 to $400 \mathrm{~m}$. Ni et al. [4] introduces the broadcast storm problem in mobile ad hoc network. The focus is on broadcast problem in MANET and broadcast storm caused by flooding. Five schemes namely Probabilistic, Counter based, Distance based, Location based and Cluster based scheme. The performance were measured through simulator which was developed in IEEE Standard 801.11 is referenced to simulate CSMA/CA behavior among hosts. Gandhi et al. [5] shows that minimizing broadcast latency in wireless network is NP complete and then present an approximation algorithm for one-to-all broadcasting. This algorithm simultaneously achieves a constant approximation both for latency as well as number of retransmissions. However, the approximation guarantee for the latency of their algorithm is greater tan 400. Huang et al. [6] obtained a 16 approximation algorithm for one-to-all broadcasting problem. It also present an algorithm with latency at most $\mathrm{R}+\mathrm{O}(\log \mathrm{R})$ where $\mathrm{R}$ ia maximum Euclidean hop distance from source to any node. However, the hidden constant in $\mathrm{O}(\log \mathrm{R})$ is not small.

Peng et al. [7] introduces the new broadcast approach that can efficiently reduce broadcast redundancy in mobile wireless network. It presents a scalable broadcast algorithm for message broadcast. The algorithm and flooding in the network simulator ns-2 and evaluate their performance through simulation. Peng et al., Sucec et al. [8][9] presents an efficient broadcast protocol for mobile ad hoc network. The proposed protocol called AHBP can relieve mobile nodes from the broadcast storm problem arising from flooding.

Stojmenovic et al. [10] propose the dominating sets and neighbor elimination based broadcasting algorithm in wireless network. The algorithm enhanced by neighbor elimination scheme and highest degree key, provides reliable broadcast with $<=53$ percent of node retransmission for all average degree d. Critical $\mathrm{d}$ is around 4 with $<=48$ percent for $<=3,<=40$ percent for $\mathrm{d}>=10$ and $<=20$ percent for $d>=25$. Orecchia et al. [11] presents localized techniques for broadcasting in wireless sensor network. Main aim has been to design solutions which only require local (one -hop neighborhood) knowledge, have low complexity, low overhead, and result in low energy consumption, low network load and high reliability. Salama et al. [12] propose the delay-constrained minimum spanning tree problem. Basagni et al. [13] present a mobility transparent broadcast scheme for mobile multihop radio networks. In this scheme, nodes compute their transmit times once and for all in the beginning. They provide two schemes with bounded latency. These schemes have approximation factors which are linear and polylogarithmic in the number of network nodes. Tiwari et al. [14] consider the one-to-all broadcast problem in 3D space. Mahjourian et al. [15] presents an approximation algorithm when both interference range and carrier sensing range are larger than transmission range. Hung et al. [16] provide centralized and distributed algorithms for broadcasting and experimental study their algorithms with respect to collision free delivery, number of transmission and broadcast latency. Williams et al. [17] survey many wireless broadcast protocols discussed above. They provide a neat characterization and experimental evaluation of many of these protocols under a wide range of network conditions.

\section{PROPOSED MODEL}

\subsection{Network Model}

The Model considered is wireless network of nodes for secure data storage over distributed node. The network consists of number of nodes. The nodes present in the network are all in the communication range of each other. The network does not have any static infrastructure. Consider a network model using directed graph $\mathrm{G}=(\mathrm{V}, \mathrm{E})$. $\mathrm{V}$ represents node and $\mathrm{E}$ is link between nodes. The nodes in $\mathrm{V}$ are in the plane. Each node has a transmission range. In this network the source node communicates with all other nodes via broadcasting mechanism. According to availability of network MAP database the data can be distributed over the wireless node for storage purpose. The medium of communication is wireless so whenever a node transmits a message all the neighbor nodes listen to the message. If node simultaneously listen message from two transmitters then it is called as a collision at the receiver node. A node listen a message from only one transmitter at a time then it is called as message without collision.

\subsection{System Architecture}

The system architecture for the secure data storage over distributed node system has seven distinct modules.

- Observer

- Data Storage Management

- XML Mentor

- Broadcast \& Schedule

- File Handler

- Sender/ Receiver

- Encryption/ Decryption 


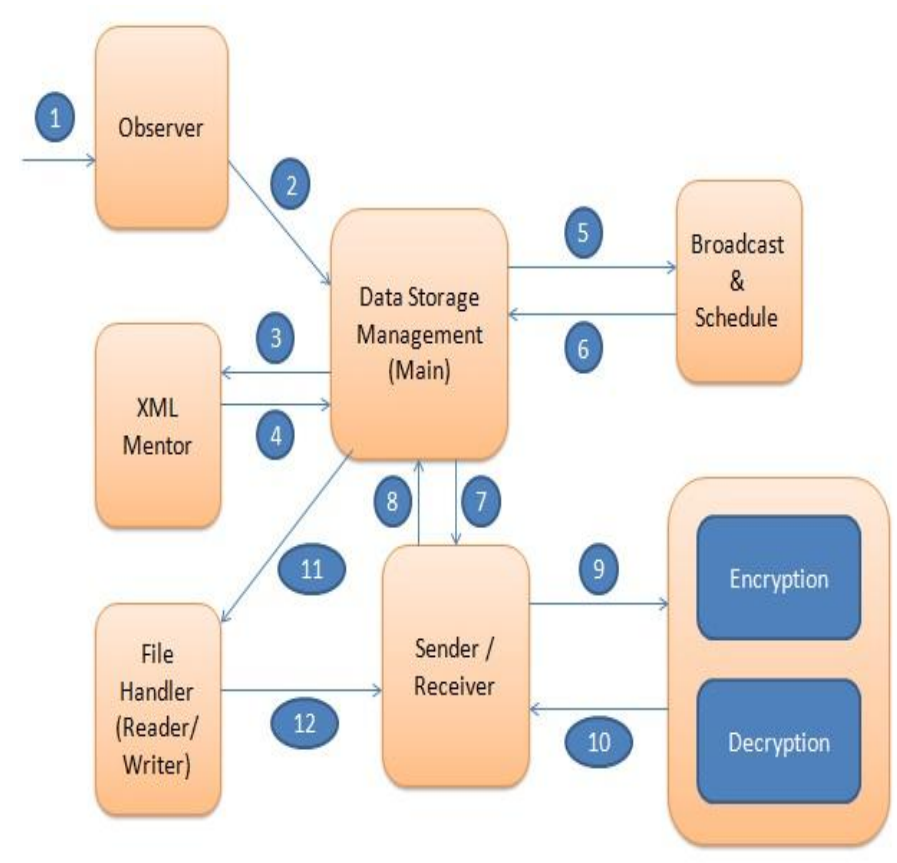

Fig -1: A secure data storage architecture system

1) Observer: Observer module continuously observers in the system. Any change occurred in the system, observer immediately inform to the main data storage module.

2) Data Storage Management: The data storage management component is main module of the system which performs all the operations of the system. It keeps control on the working of other modules.

3) XML Mentor: XML mentor maintain the XML versions of the every new created files. Also it updates the XML version of data files as and when required. If any node in the network is fails then with the help of XML versions system can create backup replica of the data on that failed node .

4) Broadcast and Schedule: It is the backbone component of system through which a broadcast tree is constructed from the data that is available from data storage management. Also it creates schedule for broadcast and broadcast the data according to schedule.

5) File Handler: It handles the read and write request that arrives from the various nodes that are available in the network. As per request it performs the read and writes operation and change the data file.

6) Sender \& Receiver: The main operation of this component is to send and receives the data file source node to destination node. It maintains two queues for read and write request that arrives from various nodes

7) Encryption / Decryption: This module mainly used for the data security before broadcasting data, data can be encrypted by using MD 5 encryption algorithm. Through this module the data can be transferred securely from source to destination.

\subsection{Broadcasting}

Broadcasting is one of major mechanism in wireless network. In this mechanism the source node communicates with another nodes through data broadcast. The system can work in four different ways such as:
1) Single Source Single Message (SSSM): In this broadcast technique there is only one source node which sends the data to all other nodes which are present in the network. At a time the source node can transmit single message to all other nodes.

2) Single Source Multiple Messages (SSMM): In this broadcast technique there is only one source node which sends the data to all other nodes which are present in the network. The source node has a capability to send multiple messages to all other nodes.

3) Multiple Source Single Messages (MSSM): In this broadcast technique all nodes of network can work as source node and each can communicate with each other's through data messages, but node can send single message to the all other nodes.

4) Multiple Source Multiple Messages (MSMM): In this broadcast technique all nodes of network can work as source node and each can communicate with each other's through data messages, but node can send multiple message to the all other nodes.

\subsection{Mathematical Model}

1. Let $(\mathrm{G}=(V, E) \mathrm{s})$

Where

$V=\left\{v_{1}, v_{2}, v_{3}, \ldots \ldots v_{n}\right\}$ set of vertices (Nodes)

$E=\left\{e_{1}, e_{2}, e_{3}, \ldots \ldots e_{n}\right\}$ set of edges

(Connections)

$s=\left\{s_{j} \mid s_{j}\right.$ is the source of message $\left.j\right\}$

$M=\{1,2, \ldots \ldots \ldots \ldots \ldots, \mathrm{m}\}$ set of messages

\section{Construct}

2. $\mathrm{V}=(\mathrm{P}, \mathrm{S})$

Where

$P=\left\{p_{1}, p_{2}, p_{3}, \ldots \ldots p_{n}\right\}$ set of primary nodes

$S=\left\{s_{1}, s_{2}, s_{3}, \ldots \ldots s_{n}\right\}$ set of secondary nodes

3. Create Schedule

4. Secure Data

5. Broadcast Message

\section{CONCLUSIONS}

This Paper gives an approximate algorithm which uses one to all and all to all data broadcast mechanism. It presents a simple and efficient way for collision free broadcast. The algorithm is also used for reducing the number of retransmission of messages in the network that results in optimal use of network bandwidth.

The future work is to extend the algorithms defined for use with dynamic topologies. As to maintain a broadcast tree in dynamic topology is very complex.

\section{ACKNOWLEDGEMENTS}

The authors would like to express heartfelt gratitude towards the people whose help was very useful to complete this dissertation work on the topic of "Distributed Data Storage using Broadcast Algorithm."

It is great privilege to express sincerest regards to P.G. 
Guide, P.G. coordinator as well as H.O.D., Prof. T. J. Parvat, for his valuable inputs, able guidance, encouragement, whole-hearted cooperation and constructive criticism throughout the duration of this work.

\section{REFERENCES}

[1] R. Gandhi, Yoo-Ah Kim, S. Lee, J. Ryu, Peng-Jun Wan "Approximation Algorithm for Data Broadcast in Wireless Networks", IEEE Trans. On Mobile Comp., Vol. 11,no. 7, pp. 1237-1248, July 2012.

[2] R. Gandhi, S. Parthasarathy, A. Mishra "Minimizing Broadcast Latency Redundancy in Ad Hoc Networks".

[3] C. Ho, K. Obraczka, G. Tsudik, and K. Viswanath, "Flooding for Reliable Multicast in Multi-Hop Ad Hoc Networks", Proc. Third Intl Workshop Discrete Algorithms and Methods for Mobile Computing and Comm., pp.64-71, 1999.

[4] S.-Y. Ni, Y.-C. Tseng, Y.-S. Chen, and J.-P. Sheu, "The Broadcast Storm Problem in a Mobile Ad Hoc Network", Proc. ACM/IEEE MobiCom, pp. 151-162, 1999.

[5] R. Gandhi, A. Mishra, and S. Parthasarathy, "Minimizing Broadcast Latency and Redundancy in Ad Hoc Networks", IEEE/ACM Trans. Networking, vol.16, no. 4, pp. 840-851, Aug. 2008.

[6] S. Huang, P. Wan, X. Jia, H. Du, and W. Shang, "Minimum-Latency Broadcast Scheduling in Wireless Ad Hoc Networks", Proc. IEEE INFOCOM, pp. 733-739, 2007.

[7] W. Peng and X.-C. Lu, "On the Reduction of Broadcast Redundancy in Mobile Adhoc Networks", Proc. ACM MobiHoc, Aug. 2000.

[8] W. Peng and X. Lu, "AHBP: An Efficient Broadcast Protocol for Mobile Adhoc Networks", J. Science and Technology, vol. 16, pp. 114-125, 2000.

[9] J. Sucec and I. Marsic, "An Efficient Distributed Network-Wide Broadcast Algorithm for Mobile Adhoc Networks", technical report, Rutgers Univ.,2000.

[10] I. Stojmenovic, M. Seddigh, and J. Zunic,'Dominating Sets and Neighbor EliminationBased Broadcasting Algorithms in Wireless Networks", IEEE Trans. Parallel and Distributed Systems, vol. 13, no. 1, pp. 14-25, Jan. 2002.

[11] L. Orecchia, A. Panconesi, C. Petrioli, and A. Vitaletti, "Localized Techniques for Broadcasting in Wireless Sensor Networks", Proc. Joint Workshop Foundations of Mobile Computing (DIALMPOMC04), pp. 41-51, 2004.

[12] H.F. Salama, D.S. Reeves, and Y. Viniotis, "The Delay-Constrained Minimum Spanning Tree Problem", Proc. IEEE Second Symp. Computers and Comm., pp. 699-704, 1997.

[13] S. Basagni, I. Chlamtac, and D. Bruschi,"A Mobility-Transparent Deterministic Broadcast Mechanism for Ad Hoc Networks", IEEE/ ACM Trans. Networking, vol. 7, no. 6, pp. 799-807, Dec. 1999.
[14] R. Tiwari, T.N. Dinh, and M.T. Thai, "On Approximation Algorithms for Interference-Aware Broadcast Scheduling in 2D and 3D Wireless Sensor Networks", Proc. Fourth Intl Conf. Wireless Algorithms, Systems, and Applications (WASA 09), pp. 438-448, 2009.

[15] R. Mahjourian, F. Chen, R. Tiwari, M. Thai, H. Zhai, and Y. Fang, "An Approximation Algorithm for Conflict-Aware Broadcast Scheduling in Wireless Ad Hoc Networks", Proc. ACM MobiHoc, pp. 331340, 2008.

[16] P.-K. Hung, J.-P. Sheu, and C.-S. Hsu, "Scheduling of Broadcasts in Multihop Wireless Networks", Proc. European Wireless, 2002.

[17] B. Williams and T. Camp, "Comparison of Broadcasting Techniques for Mobile Ad Hoc Networks", Proc. ACM MobiHoc, pp. 194-205, 2002.

[18] P. Bateman and P. Erdo s, "Geometrical Extrema Suggested by a Lemma of Besicovitch", the Am. Math. Monthly, vol. 58, pp. 306- 314, May 1951.

[19] C. Florens and R. McEliece, "Packets Distribution Algorithms for Sensor Networks", Proc. IEEE INFOCOM, pp. 1063-1072, 200, J. Breckling, Ed., The Analysis of Directional Time Series: Applications to Wind Speed and Direction, ser. Lecture Notes in Statistics. Berlin, Germany: Springer, 1989, vol. 61.

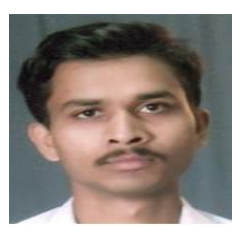

\section{BIOGRAPHIE}

Prashant S. Sadaphule received B.E. In Computer Engineering from Computer Department of K.B.P. College of Engineering \& Polytechnic, Satara from Shivaji University,Kolhapur (2008). Currently he is pursuing M.E. In Computer Engineering from STES's Sinhgad Institute of Technology, Lonavala. He is also working as an Assistant Professor in the Department of Computer Engineering in AISSMS IOIT, Shivajinagar, Pune. His area of interest is Mobile and Wireless Network 